\title{
Cooperative Development of Academic Journals and Discipline Construction in Colleges and Universities Under the "Double First-Class" Strategy
}

\author{
Shunshan Wang ${ }^{1, *}$ \\ ${ }^{1}$ The Editorial Department for Academic Journal, Nanjing University of Finance \& Economics, Nanjing, Jiangsu \\ 210003, China \\ *Corresponding author. Email: $9120121030 @ n u f e . e d u . c n$
}

\begin{abstract}
The purpose of the work is to find out the collaborative development path of academic journals and discipline construction in colleges and universities. The quantity and quality of academic journals are important indicators that reflect the development level and maturity of the discipline. Synergetic development of both sides is conducive to the realization of "Double First-class" strategic goals. In this article, we discuss the current research situation of academic journals and discipline construction in colleges and universities, in the context of the "Double First-class" strategy. We first provide the development opportunities and challenges they experience and then analyze the interaction between discipline construction and academic journals of colleges and universities. Importantly, on the basis of the perspective of university academic journals, we propose four specific paths to strengthen the research integrity, namely: brand collaboration, talent team collaboration, quality and efficiency collaboration, and platform synergy. Taken together, we believe this work can better serve the country's "Double First-class" construction.

Keywords: double first-class, colleges and universities, academic journal, coordinated development
\end{abstract}

\section{INTRODUCTION}

In January 2017, with the approval of the State Council, the Ministry of Education, the Ministry of Finance, and the National Development and Reform Commission jointly issued the Interim Measures for Coordinating the Development of World-class Universities and World-class Disciplines (hereinafter, "Double First-class"). The overall goal of "Double First-class" construction is to speed up the construction of first-class universities and first-class disciplines. The specific tasks are to train top-notch innovative talents, build a team of first-class teachers, improve the level of scientific research, pass on the fine culture of innovation, and promote the transformation of achievements. Ultimately, China expects to realize its goal of strengthening its higher education, enhancing its comprehensive strength and international competitiveness. Discipline construction is an important index that measures the development of high-quality colleges and universities and is indispensable to improving the quality of higher education. Academic journals are an important platform for the display of scientific research achievements of colleges and universities, a powerful starting point for discipline construction, and an important front for training talents of discipline construction. The academic level and editing quality of academic journals reflect the height of discipline construction and the maturity of higher education development. The "Double First-class" strategy suggests new, higher requirements for discipline construction and development of academic journals. In this context, answers to the following questions would contribute to the literature: What are the development opportunities and challenges faced by academic journals and discipline construction in colleges and universities? What is the interactive relationship between them? How can full play be given to the role of academic journals in discipline construction? What are the paths of collaborative development between academic journals and discipline construction?

\section{DEVELOPMENT OPPORTUNITIES AND CHALLENGES FOR ACADEMIC JOURNALS AND DISCIPLINE CONSTRUCTION}

The "Double First-class" strategy brings not only new development opportunities for academic journals and discipline construction in colleges and universities but also challenges. 


\subsection{The "Double First-class" Strategy Promotes the Strength and Level of Colleges and Universities and the Development of Academic Journals}

The construction of "Double First-class" has undoubtedly provided vigor and vitality to the development of colleges and universities. These institutions will take this opportunity to scientifically optimize their layout; identify their industries and school characteristics; speed up the pace of discipline construction; give full play to the leading, radiating role of superior disciplines; and increase the training of subject talents to transform and develop schools. The practice has proved that the stronger the comprehensive strength of a university, the higher the development platform of academic journals and the greater its influence. At present, most academic journals of colleges and universities are named after university journals and exist in the form of editor departments and do not have an independent legal personality. These journals' development mainly relies on the support of the university's funds, discipline construction achievements, and talent resources. The strength and level of the university will make a breakthrough in the construction of "Double First-class", inevitably providing development opportunities to the academic journals of the university. This requires the school to attach importance to journals, rely on journals, increase the investment in the personnel and financial resources of journals, and give full play to the carrier function of journals in spreading advanced culture and displaying high-level scientific research achievements, to increase the benefits of journals for researchers, teachers, and students of universities.

\subsection{The "Double First-class" Strategy Aims to Improve the Quality of Academic Journals in Universities}

"Double Leading" the implementation of the strategy to promote universities to develop their discipline characteristic and superiority is the key subject, and cultivating advantage disciplines is an inevitable requirement to achieve the goal of the service function the university attaches importance to and take full advantage of journals. Additionally, journals set up academic exchanges and discussion platforms for experts and scholars. Through the reconstruction and reorganization of the periodical columns, the column "instead of" changes to "small and exquisite," reducing weak disciplines, setting up columns corresponding to our school's characteristic subjects. Therefore, the high level scientific research achievements of the first-class disciplines in our university can be spread and transformed rapidly [1]. As an important part of the construction of colleges and universities, academic journals should be an opportunity for development and research. Additionally, journals should play a larger role in talent cultivation, discipline construction, social services, and international development, and then increasing the popularity of the school.

\subsection{The Subject Construction Evaluation Index System Brings Challenges to the Development of Domestic Academic Journals}

As an important platform for discipline construction, scientific research team training, and cultural publicity, academic journals of colleges and universities must adhere to the characteristics of individualized running journals and condensing talents while playing the role of achievement display and academic communication. At present, most of the indexes in the evaluation system of discipline construction are quantified. As an important indicator, scientific research mainly uses the number of published articles as the standard, which may easily lead to individuals misunderstanding discipline construction, believing that the achievement of discipline construction is equivalent to the number of published academic papers and papers, especially those published in international journals. The achievement of discipline construction is equivalent to the publication of academic papers and quantity, especially in international journals. Teachers blindly pursue the publication of foreign journals for professional title evaluation and promotion, resulting in the outflow of many excellent domestic scientific research achievements. The lack of high-quality manuscript sources has affected and challenged the development of domestic academic journals [2]. China has no advantages in quantity and quality of academic journals over those in developed countries. The single-minded pursuit of the quantity of journal publications leads to teachers not focusing on rigorous scholarship, hindering the improvement in the quality of academic journals in China's universities.

\subsection{The "Double First-class" Strategy Requires the Innovation Development of Academic Journals of Colleges and Universities}

The core requirement of the "Double First-class" strategy is "Chinese Characteristic, world First-class." The construction of First-class universities requires that universities change their development concept, and on the basis of the construction of First-class disciplines, they absorb the world's most advanced educational methods, make plans for the construction of the school, comprehensively improve the level of talent training and innovation ability, and realize leapfrog development and surpass guidance. The construction of First-class disciplines requires colleges and universities to focus on the construction of superior disciplines; use relevant disciplines as support; explore the characteristic development path; and build a coordinated, sustainable discipline system. Journal development must propose a higher request; change the traditional, step-by-step, passive 
service in the development of the discipline of the concept; combine the goals and tasks with the strategy of the "Double Top" innovation concept of academic journals of colleges and universities' development; think deeply of how to expand; complete the journal columns' contributions to planning and improving the quality of the redaction; and know how to support discipline construction and realize innovation and development to improve academic research. The "Double First-class" strategy suggests higher requirements for the development of academic journals of colleges and universities. The construction of First-class disciplines must have first-class thoughts, and the development mode of academic journals is in urgent need of transformation [3].

\section{THE INTERACTIVE RELATIONSHIP BETWEEN THE DEVELOPMENT OF ACADEMIC JOURNALS AND DISCIPLINE CONSTRUCTION}

Subject construction in colleges and universities and the development of academic journals depend on and complement each other. It is an inevitable requirement for improving the teaching level and scientific research strength of colleges and universities under the new situation, to make satisfactory use of and manage the relationship between them.

\subsection{Academic Journals of Colleges and Universities Promote Discipline Construction}

\subsubsection{Displaying academic achievement and servicing teachers and students for scientific research}

Through publishing excellent academic articles in various disciplines, academic journals of colleges and universities provide a platform for academic exchanges and discussion between teachers and students, and reflect their scientific research ability. The content of high-quality academic journals is highly innovative, the scope of information dissemination is wide, and the audience of knowledge is large. Teachers, students, and researchers of scientific research institutions can obtain in a timely manner the academic research results of universities and colleges by subscribing to academic journals of universities and mastering the research trends of various research fields. Comprehensive academic journals of colleges and universities display the innovations of different disciplines, enabling researchers to go to the forefront of accumulating multi-disciplinary knowledge in the process of reading. At present, an increasing number of research projects can't be completed by the knowledge accumulation of a single discipline, and the thinking mode and research method of multiple disciplines are becoming increasingly competitive in the field of scientific research. Academic journals of colleges and universities play a promoting role in cultivating researchers' multi-disciplinary perspectives and scientific research ability in interdisciplinary fields.

\subsubsection{Speeding up the talent training and promoting exchanging}

Most of the contributors to academic journals of colleges and universities are teachers, students, and researchers in scientific research institutions. Academic journals of colleges and universities promote the construction of teachers and top innovative talent in colleges and universities in two aspects. On the one hand, academic journals of colleges and universities are the platform for most young researchers to publish academic achievements and for discovering, supporting, and cultivating new academic staff. The academic achievements of many young researchers are retrieved, known, and recognized by the same industry through academic journals of colleges and universities, which plays a positive role in discovering academic talent and stimulating the academic enthusiasm of young researchers. On the other hand, the construction of "Double Top" is one of the key goals of personnel training and team building and allows increasingly fierce competition between talent in universities and colleges and that of academic journals of colleges and universities by selecting the CEC, pooling reviewing experts, and forming the authors to strengthen the communication with First-class disciplines' relevant experts and scholars and exchanges and hire them for journal development to promote the school's culture, teaching, and scientific research; give them the olive branch; contribute to talent introduction in discipline construction; and provide intellectual support for the development of the school.

\subsubsection{Leading academic research and reflecting academic advantages}

The construction of "Double First-class" focuses on improving the level of scientific research; academic journals of colleges and universities further play the role of the carrier of academic exchanges by publishing cutting-edge academic theories and research. To ensure the academic quality of journals, academic journals of colleges and universities use peer review experts and professional editors from the selected topic of academic papers, the overall framework, research methods, research conclusions, and research statements' flowing aspects to provide constructive amendments, improve the quality of the quasi-published articles, and promote the teachers' and students' in colleges and universities and research institutions academic ability, and the level of scientific research has played a role. Academic journals can reflect academic advantages. As for the developing discipline, through the carrier function of academic journals, a column with the content of the discipline as the core 
should be set up to build a discipline communication platform, expand the influence of the discipline, and speed up the progress of becoming a dominant discipline. Academic journals can also be related columns by setting the advantage in the university's disciplines and creating high-quality goods and special columns to further expand the academic achievements in the competitiveness of the academic circle and achieve a solid discipline advantage.

\subsubsection{Integrating academic resources and supporting discipline innovation}

Academic journals of colleges and universities provide a platform for teachers in various research fields to exchange research achievements; understand the latest theoretical viewpoints, research methods, and development directions; and contribute to the creation of the academic atmosphere and the improvement of the scientific research level of colleges and universities. The development of the times is bound to produce new research projects that cannot be attributed to prior disciplines or those that are interdisciplinary and integrated. At present, with the advancement of the joint development of colleges and universities, the new characteristics formed after the joint development has promoted the emergence of new disciplines. The editorial department of academic journals of colleges and universities has the closest contact with teachers and researchers of colleges and universities. Propagandizing and introducing the scientific research achievements of young teachers can mobilize their enthusiasm for scientific research and call on experts and scholars of first-class disciplines to conduct academic discussion, find and cultivate talent in various disciplines in the selection process, and tap the reserve force for innovation in various disciplines.

\subsection{Discipline Construction Promotes the Development of Academic Journals in Colleges and Universities}

\subsubsection{Promoting the characteristic development of academic journals}

Teachers and students of universities and researchers in scientific research institutions tend to discuss the phenomenon of "rushing forward" when discussing key issues, which can easily lead to the homogenization of manuscript content and the monotropism of journal manuscript sources. In the context of "Double First-class" construction, the country focuses on the construction of characteristic disciplines in colleges and universities, and the academic journals of colleges and universities also promote the goal of constructing characteristic first-class disciplines, gradually forming the professional differences among journals. The establishment of specialized research directions in the discipline construction of colleges and universities lays a foundation for the characteristic and professional development of college journals[4]. In addition, the unique geographical location has created unique regional culture and regional economic characteristics, and local university journals can make use of the advantages of regional resources and disciplines to fully realize their academic research advantages [5]. College journals depend on unique geographical advantages and research on regional characteristic columns to reveal their uniqueness, thus forming the academic journal's brand. Only through characteristic development can academic journals win the competition, and they must rely on the quality of teaching and scientific research of colleges and universities. Additionally, the development of subjects is an important support for the level of scientific research

\subsubsection{Reserving high-level professional talents}

In the context of First-class discipline construction, colleges and universities contain a group of excellent scientific research teams, who can provide high-quality manuscript sources for the development of academic journals of colleges and universities and necessary talent for the development of journals, such as authors, editorial directors, and manuscript review experts. Notably, the academic journals of colleges and universities have been limited by incomplete discipline construction and imperfect discipline talents, and there were some problems, such as insufficient manuscript sources, unstable manuscript quality, homogenized research, and difficulty in innovation. In the context of "Double First-class" construction proposed by the state, the core of discipline construction is academic echelon construction, and one of the achievements of evaluation discipline construction is to cultivate and create an academic team with certain academic status and influence in China and abroad. Therefore, in the process of "Double First-class" construction, colleges and universities compete for talent to create exclusive academic echelon talent and endeavor to recruit the best academic talent, which they then use. Thus, the academic journals of colleges and universities will attempt to obtain a group of high-level, high-quality, authors for journal development and a think-tank, and the academic level and influence of journals will substantially improve.

\subsubsection{Expanding the influence of academic journals}

The influence of academic journals generally refers to the ability of journals to publish academic research results within a certain period and promote relevant academic research and application within a certain period. The construction of first-class disciplines yields first-class academic achievements for the development of academic 
journals, and the achievements of discipline construction ultimately must be demonstrated through academic journals, to transform into productive forces and promote social and economic development. Academic journals should realize the "Double First-class" construction as an opportunity, strengthen the academic guiding function of academic journals, establish the quality assurance and evaluation system of academic journals, scientifically plan the development path, and define the development ideas and construction objectives. In promoting academic research, the quality of academic journals should be continuously improved to further enhance the status and contribution of academic journals in this field. The combination of theory and practice is emphasized in the selection and planning of journal topics. While ensuring the cutting-edge innovation and characteristics of journal contents, this paper also focuses on the selection of manuscripts to solve the contradictions of economic and social foci, to effectively improve the service function and social influence of journals.

\subsubsection{Guaranteeing the development of academic journals}

The construction of "Double First-class" requires substantial human, material, and financial resources for the construction of university infrastructure and scientific research, but this is an integral part of scientific research and infrastructure construction in colleges and universities; thus, academic journals should realize the development opportunity, scientifically plan and establish the implementation path, and make effective use of the achievements of first-class discipline construction and the influence of first-class universities. Running first-class academic journals to achieve scientific development is a plan. As a main mode of self-promotion, academic journals of colleges and universities are a platform to display their scientific research level. These institutions are paying increasing attention to the role of this platform. Therefore, an urgent task is to strengthen the all-round support for academic journals, enhance the quality of discipline construction by developing first-class disciplines, and provide high-quality academic achievements for academic journals. Additionally, the conditions, office equipment, and working environments of academic journals should be improved to solve funding problems, and certain aspects, for example, the holding of academic conferences, training of editors, construction of new media of journals, and economic treatment of academic journal editors should be improved to attract talents to participate in constructing academic journals [6]

\section{THE PATH OF COLLABORATIVE DEVELOPMENT OF ACADEMIC JOURNALS AND DISCIPLINE CONSTRUCTION}

What is necessary and feasible is to maintain the positive interaction between the development of academic journals and discipline construction in colleges and universities, and this requires overall planning and scientific planning in practical work to realize the coordinated development of the two.

\subsection{Synergy of Featured Brands: Creating Characteristic Journals and Promoting the Development of Advantageous Disciplines}

At present, the diversification of the development of colleges and universities in China determines the diversification of academic journals of colleges and universities, and characteristic journals are a means of long-term survival. To stand out in diversified development, academic journals must use the characteristic methods, and the specialty and characteristic development of journals rely on the advantageous subject resources of the school.

Firstly, the "Double First-class" construction yields an opportunity for the construction of characteristic journals. Academic journals can enhance academic popularity and seek sustainable development according to the characteristics of economic and cultural development in their regions and combination with the academic characteristics of the school, thus contributing to the construction of first-class disciplines. Combined with the "Double First-class" construction goal, academic journals of colleges and universities should be based on science frontiers; clarify subject development according to the school's disciplines, advantages, selecting and planning, and targeted set columns according to the need of subject construction; build first-class international advanced main columns' characteristic columns, the brand, and the periodical characteristic of the development of the road; and promote the development of the social economy and culture.

Secondly, the human, material, and financial support from the construction of first-class disciplines should be used to further advance the role of academic journals as a platform for academic exchanges[7]. Considering the leading role of academic journals in academic research, some colleges and universities have further increased the talent and funding input of academic journals and realized the role of journals in cultivating the talent of superior disciplines, especially young and middle-aged talent. In the process of providing services for discipline construction, academic journals of colleges and universities recruit and train outstanding scientific research talent for colleges and universities by tracking and paying attention to the scientific research situation of young scholars. Additionally, they solicit 
contributions from experts and scholars in first-class disciplines to obtain high-quality resources and realize the coordinated development of academic journals and discipline construction in colleges and universities.

Thirdly, the development of academic journals and the construction of advantageous disciplines complement each other. Academic journals of colleges and universities play an important role in promoting the construction of superior disciplines: on the one hand, it cultivates scientific research talent for colleges and universities, and on the other hand, it spreads the achievement of discipline construction in colleges and universities. In addition, the dominant discipline is the value direction of soliciting contributions for academic journals of colleges and universities, and is an important means to build their academic brands. This endeavor requires universities to pay equal attention to teaching and scientific research in the development process and absorb high-quality teachers and high-quality students to provide talent support for the development of journals [8].

\subsection{Synergy of Talent Team: Strengthening the Construction of the Editorial Team and Reserving Talent for Discipline Construction}

If the academic journals of colleges and universities are regarded as the connecting tools of academic communication, the journal editors are the porters of academic achievements. The editorial staffs play a vital role in the type and quality of the deliverables. The quality of the editorial team reflects the level and development potential of the journal to a certain extent. First, editorial staff should enhance the "Four Consciousnesses" in their work and resolutely achieve the "Two Maintenances." It should adhere to the socialist direction and the development of socialist culture; have Chinese characteristics; promote the economy of the principle, responsibility and the mission, and their innovative work ideas; consistently explore new channels and the periodical development of editing work with enthusiasm and willingly support the workers in the spread of the academic achievements. Then, the staff of the editorial department should take the initiative to strengthen communication and exchange with outstanding researchers and establish a good cooperative relationship by attending journals' and academic conferences. Traditional editors only passively put their energy into contributions from nature and seldom actively contact and encourage the excellent researchers around them to contribute; thus, academic journals' means of securing excellent sources and contributors become limited. In the long run, the low quality of manuscripts hinders the development of academic journals and the whole discipline.

Finally, the development of new media has changed the production, dissemination, and evaluation methods of academic knowledge. Thus, it is imperative to integrate the resources of journals with the help of media integration skills, which suggests new requirements for the construction of academic journal talent in colleges and universities and is embodied in the three aspects: workers are required to set up the publication of the media convergence development concept; build up talent-attracting, training, and incentive mechanisms; and pay attention to the comprehensive-ability training of workers ability to understand new media technology, the part-time editor function, and how to strengthen communication editorial skills training by applying the internationalization development view and so forth. [9]

\subsection{Synergy of Quality and Efficiency: Improving the Editing Efficiency of the Editorial Department and Guaranteeing Discipline Construction}

To win the competition, academic journals of colleges and universities must ensure academic quality.

To achieve this objective, firstly, they should strictly implement the system of three reviews and three schools, effectively use the anonymous review system to check and improve manuscript quality, and reduce the review time and improve the efficiency of review.

Secondly, they should promote external expert database construction; integrate internal and external resources, multi-channel and omni-directional, to invite scholars in the fields of various disciplines, increase the number of experts in the database, and optimize expert team quality; match the subject direction and research content with a high academic level and a strong sense of responsibility of outside experts; and pay attention to updates and strengthening responsibility consciousness, guaranteeing the journal's quality control.

Thirdly, they should realize the discipline advantages to expand manuscript sources, effectively absorb research results of advantageous disciplines of the university, and solicit manuscripts from experts and scholars of relevant disciplines. In this process, it is also necessary to inherit and develop advantageous disciplines of the university. Finally, also necessary is to innovate the procedures for soliciting contributions for journals, always pay attention to the most relevant issues and discipline trends, and attract high-quality contributions by convening experts and scholars in related fields by holding discipline seminars, organizing essay contests, and publishing on the year's hot topics.

\subsection{Platform Synergy: Accelerating the Integrated Development of New Media of Journals and Innovating the Platform of Discipline Construction}

As the display platform of frontier scientific research achievements, the effect of timeliness on the development of academic journals is self-evident. Therefore, in the process of the development of university journals, the 
integration of new media technology to disseminate scientific research results is an inevitable trend of The Times. Firstly, academic journals of colleges and universities should uphold the principle of "content is king" and fully realize the convenience and interest advantages of new media communication to realize their sustainable development in innovative communication.

Secondly, they should broaden the channels of new media communication. At present, university academic journals can achieve effective communication between journal editors and journal authors through email, websites, WeChat official accounts, and other forms. In addition, colleges and universities can accelerate the integration of new media by constructing PC periodical home pages and mobile client terminals. Through strengthening the construction of new media in journals, improving authors' contributions, and innovating the channels of publication and distribution, the editing and reviewing of the efficiency of the editorial department can be improved, and the dissemination speed of subject knowledge can be accelerated. However, with the surge of WeChat users in recent years, academic journals of colleges and universities can publish in timely manner dynamic information related to academic journals, update disciplinary research results, and realize the interaction between editorial departments and authors by opening the official WeChat public accounts of academic journals. This new media communication method not only has the advantage of covering web page communication but also can realize online interactions among the editors, readers, and journal authors of academic journals of colleges and universities, to fulfill readers' requirements for the exploration of subject knowledge.

Thirdly, they should integrate the internal and external resources of universities and periodical editorial departments and promote the benign development of university disciplines and journals by strengthening the exchanges and cooperation with other universities and periodical departments to exchange needed goods, complement each other's advantages, and achieve win-win results.

In summary, academic journals and discipline construction in colleges and universities are mutually influencing and promoting each other. The "double top" strategy of colleges and universities (especially the location advantage and advantage of colleges and universities) and subject construction yielded opportunities and challenges, that is, the development of academic journals and the opportunity for academic journals of colleges and universities to improve scientific research platforms, attract more experts and scholars to the school discipline, and contribute to the development of the discipline of the school. The academic journals should understand the policy, talent, and resource conditions due to "Double First-class" construction, strengthen their construction, contribute to the realization of the national "Double First-class" strategic goal, and realize the coordinated development of discipline construction.

\section{CONCLUSION}

The implementation of the "Double First-class" strategy is conducive to further enhancing the comprehensive strength and international competitiveness of China's higher education, and also puts forward higher requirements for discipline construction and academic journals. Academic journals and the discipline construction promote each other, it requires overall planning and scientific planning in practical work to realize the coordinated development of the two. There are four paths to choose: creating characteristic journals and promoting the development of advantageous disciplines; strengthening the construction of the editorial team and reserving talent for discipline construction; improving the editing efficiency of the editorial department and guaranteeing discipline construction; accelerating the integrated development of new media of journals and innovating the platform of discipline construction.

\section{ACKNOWLEDGMENT}

We would like to thank editors and anonymous referees for useful comments and suggestions. We acknowledge financial support from the Jiangsu Provincial University Philosophy and Social Science Research Fund Project (NO. 2018SJA0258). Educational Reform Project of Nanjing University of Finance and Economics (NO GJY1863).

\section{REFERENCES}

[1] X. Hao, C. G. Liu, Y. S. Yang, "Problems of university journals serving double first class construction and the corresponding countermeasures: taking Liaoning province as an example, " Chinese Journal of Scientific and Technical Periodicals, vol. 29, pp. 882-887, September 2018. (In Chinese)

[2] Y. Y. Zheng, Y. W. Li, X. F. Mo, and S. F. Mao, "Opportunities and challenges of college journals in the construction of 'Double First-Class' university," Acta Editologica, vol. 29, pp. 160-162, April 2017. DOI:10.16811/j.cnki.1001-4314.2017.02.018. (In Chinese)

[3] W. M. Zeng, W. Li, Z. B. Zhao, "Practice and thinking of college journal service discipline construction," Journal of East China University of Technology(Social Science), vol. 29, pp. 195-197, June 2010. (In Chinese)

[4] H. J. Yang, Y. Q. Xu, "Analysis on the Relationship between university journals and discipline construction in universities," Acta Editologica, vol. 26, pp. 102-104, December 2014 
DOI:10.16811/j.cnki.1001-4314.2014.s1.040. (In Chinese)

[5] Z. B. Liu, C. P. Du, J. Xue, "Study on the relationship between university journal and subject construction," Journal of China Three Gorges University (Humanities \& Social Sciences), vol. 27, pp. 104-107, October 2005. (In Chinese)

[6] G. Z. Yang, Y. J. Liu, “Academic journals and First-class discipline construction in universities: leading, promoting and developing," Publishing Journal, pp. 19-22, May 2018.

DOI:10.13363/j.publishingjournal.2018.03.003. (In Chinese)

[7] S. G. Liu, “ The characteristic development of humanities and social sciences journals in the New Era and on the role of journals in the construction of philosophy and social sciences with Chinese characteristics," Seeker, pp. 4-13, October 2018. DOI:10.16059/j.cnki.cn43-1008/c.2018.05.001. (In Chinese)

[8] H. F. Gao, "The role of university Sci-Tech periodicals in promoting the construction of 'Double First-class',' Journal of Northwest Minzu University (Natural Science), pp. 91-95, April 2018. DOI:10.14084/j.cnki.cn62-1188/n.2018.02.019. (In Chinese)

[9] S. S. Wang, "The Construction of talent team of academic journals under the background of New Media," Journal of Nantong University (Social Sciences Edition), pp. 150-154, August 2018. (In Chinese) 